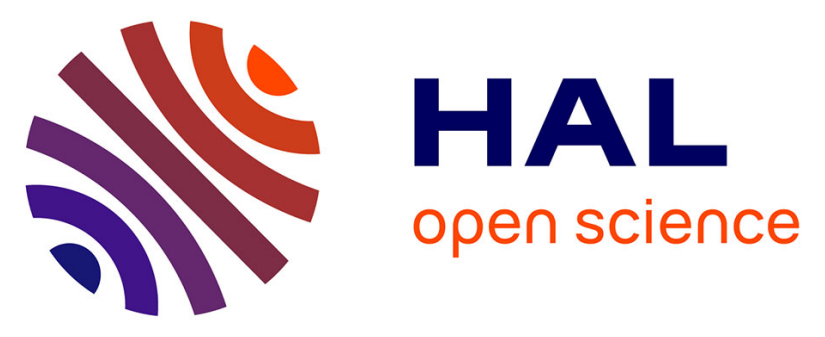

\title{
Novel mutations in the bone morphogenetic protein 6 gene in patients with iron overload and non-homozygous genotype for the HFE p.Cys282Tyr mutation
}

Aline Morgan Alvarenga, Nathália Kozikas da Silva, Paula Fernanda Silva Fonseca, Theo G M Oliveira, Jacilene Barbosa da Silva Monteiro, Rodolfo Delfini Cançado, Flavio Augusto Naoum, Carla Luana Dinardo, Pierre Brissot, Paulo Caleb Junior Lima Santos

\section{- To cite this version:}

Aline Morgan Alvarenga, Nathália Kozikas da Silva, Paula Fernanda Silva Fonseca, Theo G M Oliveira, Jacilene Barbosa da Silva Monteiro, et al.. Novel mutations in the bone morphogenetic protein 6 gene in patients with iron overload and non-homozygous genotype for the HFE p.Cys282Tyr mutation. Blood Cells, Molecules and Diseases, 2020, 84, pp.102444. 10.1016/j.bcmd.2020.102444 . hal-02864471

HAL Id: hal-02864471

https://hal-univ-rennes1.archives-ouvertes.fr/hal-02864471

Submitted on 15 Jun 2020

HAL is a multi-disciplinary open access archive for the deposit and dissemination of scientific research documents, whether they are published or not. The documents may come from teaching and research institutions in France or abroad, or from public or private research centers.
L'archive ouverte pluridisciplinaire $\mathbf{H A L}$, est destinée au dépôt et à la diffusion de documents scientifiques de niveau recherche, publiés ou non, émanant des établissements d'enseignement et de recherche français ou étrangers, des laboratoires publics ou privés. 


\section{Novel mutations in the bone morphogenetic protein 6 gene in patients}

with iron overload and non-homozygous genotype for the $\mathrm{HFE}$

\section{p.Cys282Tyr mutation}

Aline Morgan Alvarenga ${ }^{1}$, Nathália Kozikas da Silva ${ }^{1}$, Paula Fernanda Silva Fonseca ${ }^{1}$, Theo G M Oliveira ${ }^{2}$, Jacilene Barbosa da Silva Monteiro ${ }^{1}$, Rodolfo Delfini Cançado ${ }^{3}$, Flavio Augusto Naoum ${ }^{4}$, Carla Luana Dinardo ${ }^{5}$, Pierre Brissot ${ }^{6}$, Paulo Caleb Junior Lima Santos ${ }^{1 *}$

a.cmorgan@hotmail.com;nathalia.kozikas@unifesp.br;paulafonseca@usp.br;theo.grem en@usp.br;jacybiologa@ hotmail.com;rdcan@uol.com.br;fnaoum@hotmail.com;caludi nardo@gmail.com;pierre.brissot@gmail.com;paulo.caleb@unifesp.br

${ }^{1}$ Department of Pharmacology - Escola Paulista de Medicina, Universidade Federal de São Paulo (EPM-Unifesp), São Paulo, Brazil.

${ }^{2}$ Laboratory of Genetics and Molecular Cardiology, Heart Institute (InCor), University of São Paulo Medical School, São Paulo, Brazil.

${ }^{3}$ Hematology Division, Santa Casa de São Paulo Medical School.

${ }^{4}$ Academia de Ciência e Tecnologia, São José do Rio Preto, Brazil.

${ }^{5}$ Fundação Pró-Sangue, Hemocentro de São Paulo, São Paulo, SP, Brazil; Universidade de São Paulo (USP), São Paulo, SP, Brazil.

${ }^{6}$ Institut NuMeCan, Inserm U-1241, Univ Rennes 1, Rennes, France

\section{* Correspondence:}

Paulo Caleb J L Santos, PhD

Department of Pharmacology, Escola Paulista de Medicina, Universidade Federal de São Paulo, EPM-Unifesp, São Paulo, Brazil. paulo.caleb@unifesp.br 


\begin{abstract}
Background: Five main genes are associated with hemochromatosis; however, current studies show that, in addition to these genes, others may be associated with primary iron overload (IO). One of these is the bone morphogenetic protein 6 (BMP6), which encodes a protein that modulates hepcidin synthesis and, consequently, iron homeostasis. Aim: To identify BMP6 gene pathogenic variants in patients with IO and non-homozygous genotype for the HFE p.Cys282Tyr mutation. Materials and methods: Fifty-three patients with primary IO and non-homozygous genotype for the HFE p.Cys282Tyr were selected. Subsequent bidirectional DNA sequencing of BMP6 exons was performed. Results: Two novel variants were found. One at homozygous state p.Gln158Ter (c.472C >T) was pathogenic, the other one at heterozygous state p.Val394Met (c.1180G> A) was of uncertain significance (VUS); the third variant at heterozygous state p.Arg257His (c.770G >A) has already been described and associated with IO. No BMP6 pathogenic variants that would explain iron overload phenotypes were detected in $94 \%$ of the studied patients. Conclusion: Identification of the BMP6 pathogenic variants in Brazilian patients with primary IO might contribute to the genetic understanding of this phenotype.
\end{abstract}

Keywords: Hemochromatosis, iron overload, BMP6, sequencing, non-homozygous genotype for the HFE p.Cys282Tyr. 


\section{Background}

Hemochromatosis is an autosomal recessive disease, in which iron overload is related either to a decrease in hepcidin production or to a defect in hepcidin binding to ferroportin (1-2). In most cases, hemochromatosis is associated with homozygosity for the HFE p.Cys282Tyr mutation. Although p.C282Y/p.H63D compound heterozygosity has been associated with hemochromatosis, this genotype is mainly a risk factor for slightly higher serum iron parameters and mildly increased hepatic iron stores, and it is considered insufficient to cause hemochromatosis, according with current guidelines (3). Rare cases of hemochromatosis can also be caused by pathogenic variants in other genes, corresponding to non-HFE hemochromatosis (4-8). Juvenile hemochromatosis $(\mathrm{JH})$ is type 2 , subdivided into type $2 \mathrm{~A}$, related to a pathogenic variant in the $H J V$ (hemojuvelin) gene, and type $2 \mathrm{~B}$, related to mutations in the HAMP (hepcidin) gene; type 3 is related to a pathogenic variant in the TFR2 gene; and type 4 is related to a pathogenic variant in the SLC4OAl (ferroportin) gene (9).

Some studies have reported that sequencing the five main genes related to hemochromatosis may not be sufficient to explain all cases of primary iron overload (IO) (10,11). A Portuguese group, using next generation sequencing (NGS), evaluated a panel of genes involved in iron metabolism (HFE, TFR2, HJV, HAMP, SLC4OA1 and FTL (L-ferritin)) in 87 patients with a non-classical form of hemochromatosis, and were unable, in some patients, to detect changes that could explain their phenotype, which suggests that the genetic changes possibly responsible for IO in these patients could be located in genes not addressed in this study (11). One study sequenced the HFE, $H J V$, HAMP, TFR2 and SLC4OA1 genes from 51 samples from Brazilian patients with 
primary IO. The frequency of the homozygous p.Cys282Tyr genotype in the HFE gene in this sample was significantly lower (21.6\%) than in other studies (12),

Great advances in the molecular diagnosis of hemochromatosis have already been observed, but it remains hemochromatosis cases for whom no changes are found in the main genes related to iron homeostasis. Thus, it is important to explore further genes involved in iron metabolism, in order to better understand the molecular basis of hemochromatosis). Hamdi-Rozé et al reported that rare $H F E$ variants may be a frequent cause of hemochromatosis in non homozygous p.Cys282Tyr hemochromatosis patients. BMPs (bone morphogenetic proteins) are proteins that belong to the Transforming Growth Factor Beta (TGF-beta) superfamily, which is involved in cell differentiation and cell proliferation $(13,14)$. The BMP / SMAD signalling pathway is strongly involved in systemic iron regulation through its impact on the hepcidin gene (HAMP) transcription (17 - 16). In animal models, BMP6 expression is highly related to iron homeostasis; in wild-type mice, iron-enriched diet leads to an increase in both BMP6 expression and SMAD1/5/8 phosphorylation, which was correlated with an increase in HAMP transcription $(18,19)$. In another study, it was found that mice with a deficiency in $B M P 6$ expression showed a reduction in hepcidin levels and a phenotype similar to patients diagnosed with hemochromatosis $(20,21)$. In a human study, hepcidin levels were markedly low in patients who had pathogenic variants in the BMP6 gene (22). Liver cell isolation experiments indicates that non parenchymal cells (NPCs), considering Kupffer cells (KCs), sinusoidal endothelial cells (SECs), and hepatic stellate cells (HSCs) rather than hepatocytes, are the main source of BMP6 in the liver (23). Taken together, these data suggest that BMP6 plays an important role in the regulation of hepcidin; thus, mutations in this gene may lead to iron-related disorders, as iron overload. 
Therefore, our aim was to search for $B M P 6$ gene pathogenic variants in patients with IO and non-homozygous genotype for the HFE p.Cys282Tyr mutation.

\section{Methods}

\subsection{Patients}

The study protocol was approved by the Ethics Committee (EPM-Unifesp 1233/2018 and HC-FMUSP - 4027/14/007, Brazil) and consent form was obtained from the patients prior to entering the study. Fifty-three patients were selected from hematology ambulatory clinics (Hematology Outpatient Clinic of Hospital das Clínicas - São Paulo, Outpatient Clinic of the Blood Center of Santa Casa - São Paulo, and Naoum Institute of Hematology - São José do Rio Preto) in Brazil. Inclusion criteria were: age greater than or equal to 18 years, plasma transferrin saturation (TS) $\geq 45 \%$ and serum ferritin $(\mathrm{SF}) \geq 200 \mathrm{ng} / \mathrm{mL}$ for women and $\geq 300 \mathrm{ng} / \mathrm{mL}$ for men. The exclusion criteria were patients with homozygosity for the HFE p.Cys282Tyr mutation, positive serology for hepatitis $\mathrm{C}$ or $\mathrm{B}$, alcoholic liver disease, high alcohol consumption (>20g per day), haemolytic anaemia, repeated blood transfusions, metabolic syndrome or insulin resistance not resulting from hemochromatosis (24).

\subsection{DNA extraction and genotyping}

The blood samples of the patients were collected using the BD Vacutainers System (Becton Dickinson, NJ, USA), and genomic DNA (DNAg) was extracted from the leukocytes using the QIAamp DNA Blood Mini Kit commercial extraction method (QIAGEN), in accordance with the protocol described by the manufacturer (23). HFE p.Cys282Tyr (c.845G>A; rs1800562) and p.His63Asp (c.187C >G, rs1799945) 
mutations were genotyped using Real Time Polymerase Chain Reaction (PCR 7500 Applied Biosystem real-time PCR, USA - TaqMan assays).

\subsection{Primer design, Sanger sequencing and analysis}

Eigth primer pairs were designed to explore the 7 exons of the BMP6 gene, expanding to $20 \mathrm{bps}$ of intronic regions, (supplementary Table 1). The PCR products were purified with the ExoSAP-IT reagent (GE Healthcare, NJ, USA) and the Sanger methodology was applied using the Applied Biosystems 3130 / 3130xl Genetic Analyzers, the analysis of the results in electropherogram format was carried out by the Geneious software (Biomatters).

\subsection{Data analysis}

To classify the changes found in the $B M P 6$ gene, the lowest allele frequency indicated for rare diseases (MAF <0.01\%) was used (25). Population data were consulted: EVS (Exome Variant Server), gnomAD (Genome Aggregation Database) and ABraOM (Brazilian Online Archive of Mutations). In addition, pathogenicity prediction software was used: Polyphen-2 (Polymorphism Phenotyping v2), which estimates structural and functional effects of human variants and analyses the evolutionary conservation of the affected protein. The predictions were calculated for all resulting amino acid residue substitutions in human UniProtKB proteins with the maximum CDS sequence overlap and identity. Provean (protein variation effect analyser), which seeks to impact the biological function of a protein, was used. The Provean scores are computed based on the homologs collected from a database. Thus, the prediction results may slightly vary with the protein database used and also the versions of PSI-BLAST and CD-HIT programs. Currently, the PROVEAN web server 
uses the NCBI nr database (September 2012), BLAST v2.2.24+, and CD-HIT v4.5.4. Sift (Intolerant Classification of Tolerant), which is based mainly on the evolutionary condition of the protein, uses a query string and various alignment information to predict tolerated and harmful substitutions for all positions in the query string. To obtain the result, there is a procedure with several steps, among which we have the search for similar sequences, choice of closely related strings that can share a function similar to the query string, the alignment of these chosen sequences is obtained and normalized probabilities are calculated for all possible alignment substitutions. Positions with normalized probabilities less than 0.05 are predicted to be harmful, those greater than or equal to 0.05 are predicted to be tolerated. The mutation taster that performs a series of in silico tests to estimate the impact of the variant on the protein and to determine its disease-causing potential. The changes were compared with the HGMD database (Human Gene Mutation Database) and ClinVar, which aggregate information on genomic variation and its relationship to human health, as well as articles found on Pubmed. For pathogenic variant p.Gln158Ter (c.472C> T), we also used the CADD software, which has $\mathrm{C}$-scores for all 8.6 billion possible single nucleotide variants (SNVs) of the reference genome, a selection of short insertions / deletions, as well as a few sets of large variants (e.g., gnomAD, ExAC, 1000 genomes, ESP). It also provides a simple search for SNVs and allows the scoring of short insertions / exclusions. Finally, the changes found were classified according to the ACMG (American College of Medical Genetics and Genomics) criteria (26).

\section{Results}

\subsection{Identified genetic variants}


Of the fifty-three patients, we identified three $B M P 6$ variants $(3 / 53,6 \%)$ with potential for pathogenicity. They are a novel pathogenic variant in patient \#01, a novel variant of uncertain significance (VUS) in patient \#02, and a pathogenic variant, already described, in patient \# 03. The novel pathogenic variant, at homozygous state, found in patient $\# 01$, was p.Gln158Ter (c.472C $>\mathrm{T}$, exon 1). The novel VUS, at heterozygote state, found in patient \#02, was p.Val394Met (c.1180G>A, exon 4). Patient \#03 has the pathogenic variant p.Arg257His (c.770G>A, exon 2) at the heterozygous state.

The rs2241669 polymorphism, present in exon 2, was observed in 4 patients (allelic frequency 3\%). In exon 4, the rs17557 polymorphism was found in 29 patients (17\%), and the rs 17558 in 4 patients $(3 \%)$.

No BMP6 mutation that would explain iron overload phenotypes was detected in $94 \%(50 / 53)$ of the studied patients.

\subsection{Patients data}

We enrolled fifty-three patients with mean age of $49 \pm 13,79 \%$ of males. The mean values of plasma TS and serum ferritin were $65 \pm 19 \%$, and $1,375 \pm 840 \mu \mathrm{g} / \mathrm{L}$, respectively.

Table 1 shows data of the patients. Patient \#01 was a 61-year-old Brazilian man, with TS of $82 \%$ and SF of $1,505 \mu \mathrm{g} / \mathrm{L}$. Genetic analysis for pathogenic variants in the HFE gene, p.Cys282Tyr and p.His63Asp, revealed a heterozygous genotype for both. The BMP6 sequencing revealed the homozygous genotype for the novel pathogenic variant at position p.Gln158Ter (c.472C > T). It is a nonsense mutation, and causes a premature stop codon in the protein according to the mutation taster. 
Patient \#02 is 46-year-old Brazilian man. TS was $61 \%$ and SF 1,096 $\mu \mathrm{g} / \mathrm{L}$. In addition to the sequencing of the BMP6 gene, sequencing was also performed for HFE, HJV, HAMP, TFR2, and SLC4OA1 genes, and no variants were found. The patient reported that his father, mother and two sisters were diagnosed with hemochromatosis. One of his sisters died at the age of 41 because of complications from iron overload. The BMP6 sequencing revealed heterozygosity for the novel p.Val394Met, classified as VUS (variant of uncertain significance).

A 32-year-old Brazilian man is patient \#03, with SF of $301.4 \mu \mathrm{g} / \mathrm{L}$, TS of $46.4 \%$ and secondary IO excluded. The patient reported that his father was diagnosed with hemochromatosis. Genetic analysis revealed a compound heterozygous genotype for HFE p.Cys282Tyr and p.His63Asp. The BMP6 sequencing revealed the heterozygous genotype for the pathogenic variant p.Arg257His, described in the literature (27).

\subsection{Population frequency, in silico prediction, classification according to}

\section{ACMG}

The population frequency and in silico prediction of the alterations are listed in Table 2. After evaluating the genetic results of the alterations, they were classified into levels of pathogenicity according to the ACMG criteria that we included also in Table 2.

For the alteration p.Gln158Ter (c.472C $>\mathrm{T})$ no population frequency data was found and the only in silico predictor that has information about this alteration was a mutation taster that was highlighted as a "prediction disease causing" alteration. According to the CADD tool, which is the most complete for annotating variants and can analyze nonsense variants, the program's authors suggest a cut-off of 15 to consider the change as harmful (in your case, it was 34). Another interesting point about the variant is that it is not present in gnomad. This site gathers genetic information from 
individuals selected to be "control population" (theoretically without Mendelian diseases in the family). GnomAD is the largest bank today. If we look at nonsense changes (or loss of function), we see that they only appear in heterozygosity in gnomad, and it was found in homozygosity in our analysis. According to the ACMG, it was classified as a pathogenic alteration.

The population frequency found for p.Val394Met change was $0.0821 \%$ in ABraOM and $0.05129 \%$ in GenomAD. Regarding predictors in silico: Provean proved it to be a "tolerated" alteration, PolyPhen-2 described the variant as "benign", Sift "tolerates" the variant, and the mutation taster described it as "disease causing". This change was not found in ClinVar or HGMD; according to ACMG, it was classified as a VUS type, as it did not score enough to be pathogenic or possibly pathogenic.

The p.Arg257His pathogenic variant had a population frequency of $0.4936 \%$ in $\mathrm{ABraOM}$ and of $0.1375 \%$ in GenomAD. Predictors in silico reported that: in Provean the variant is considered "Damaging", in PolyPhen-2 it is "Probably damaging", in Sift the variant is described as "deleterious" and in mutation taster it is said to be "disease causing". This change was not found in ClinVar and in HGMD. According to ACMG classification, it is a pathogenic alteration.

\section{Discussion}

In our study, we identified a known pathogenic variant (BMP6 p.Arg257His), as well as two novel changes not yet described in the literature, namely p.Gln158Ter (c.472C>T), classified as pathogenic and p.Val394Met, classified as VUS.

The latter variant, classified as VUS, does not have data of population frequency in the majority of predictors in silico. There are data that indicate that the VUS might be pathogenic. Our patient had a first-degree relative with iron overload. Nevertheless, 
according to ACMG, there was not enough score to classify it as pathogenic and, therefore, it is necessary to improve the data related to these changes described as VUS.

In 2016, Daher et al sequenced the BMP6 gene in samples from 70 patients with the IO phenotype who did not carry any genetic alteration for the major hemochromatosisrelated genes (28). In this study, three different BMP6 propeptide heterozygous pathogenic variants were identified (p.Pro95Ser, p.Leu96Pro and p.Gln113Glu). According to their analyses, these pathogenic variants showed a strong association with the IO phenotype observed in the studied patients. In addition, they also performed in vitro studies to investigate the possible functional alterations caused by the identified pathogenic variants. They showed that these pathogenic variants were able to impair both the BMP6 protein secretion and the SMAD1/5/8 signalling pathway activation, thereby leading to reduced hepcidin production by the liver. Taken together, these data suggest that BMP6 propeptide pathogenic variants may be responsible for causing IO in some patients (28). There was a strong association of heterozygosity for three different BMP6 propeptides with the IO phenotypes observed in the corresponding patients (28).

The same p.Leu96Pro alteration, previously described by Daher et al (28), was also identified by an Italian group in 2017, along with two other novel BMP6 propeptide pathogenic variants (p.Glu112Gln and p.Arg257His), in patients with IO (27). According to in silico analysis, the amino acid substitutions caused by the p. Leu96Pro and p.Glu112Gln pathogenic variants are located at key regions related to interactions between monomers, while the amino acid change caused by the p.Arg257His pathogenic variant may affect the interaction between $B M P 6$ and extracellular matrix proteins. Therefore, the data suggest that these pathogenic variants could lead to inappropriate BMP6 protein assembly, as well as affect its cellular localization. However, no experimental data have been reported yet to verify this hypothesis (27). 
While previous studies lead us to believe that $B M P 6$ propeptide pathogenic variants are associated with protein changes that can cause IO $(27,28)$, a recent study suggests that attributing the cause of the disease to these propeptide alterations should be considered with caution (29). This study evaluated three clinical cases of unexplained IO carrying the propeptide BMP6 pathogenic variant p.Gln118_Leu119insGlnGlnHis. In vitro data showed no difference in BMP6 secretion or localization between the wild type and the p.Gln118_Leu119insGlnGlnHis BMP6 alteration, indicating that this alteration does not affect the BMP6 protein function. Moreover, the functional impact prediction using bioinformatics tools confirmed the benign nature of this alteration. Thus, the authors concluded that there is no functional relationship between the BMP6 propeptide p.Gln118_Leu119insGlnGlnHis variation and IO, in contrast to what was proposed in previous studies.

The novel pathogenic variant p.Gln158Ter (c.472C> T), causes a premature stop codon in the protein, and, in our homozygote patient for this variant, the expression of the gene is expected to be totally absent. It was not possible to perform in vitro tests for documenting the loss of the BMP6 protein, but through the analysis done in the CADD software, which is a most complete tool for annotating variants and analysing nonsense variants, we found a PHRED value of 34, much higher than the cut-off value of 15 above which the change can be considered as harmful. We also used gnomad, which gathers genetic information from individuals selected to be "control population" (theoretically without Mendelian diseases in the family). GnomAD is the largest database today, and when we searched for nonsense (or loss of function) changes we found that the variant so far has not been observed in healthy individuals, and that all nonsense variants in BMP6 reported to date in control populations were at the 
heterozygous state. This reinforces the hypothesis that homozygous nonsense variants in BMP6 can lead to the observed phenotype.

Our study also found a further novel variant p.Val394Met in the BMP6 gene that was classified as VUS, whose pathogenicity is possible. The identification of novel pathogenic variants associated with IO is important to provide the basis for further research aiming at elucidating the role of this gene in the spectrum of genetic iron overload diseases.

In conclusion, identification of the BMP6 pathogenic variants in Brazilian patients with primary IO might contribute to the genetic understanding of this phenotype. 
$\# 01$ p.Gln158Ter c. $472 \mathrm{C}>\mathrm{T}$
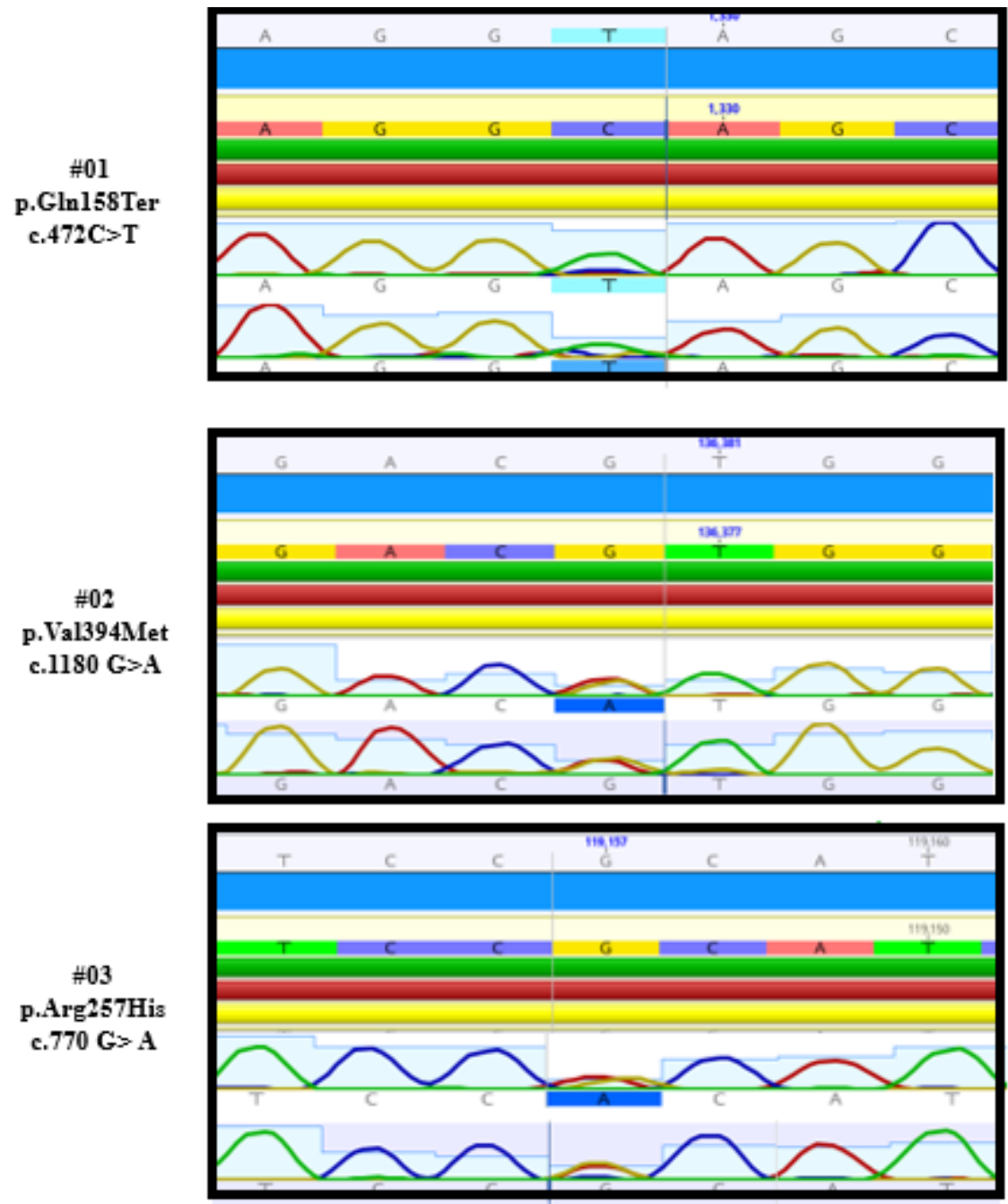

FIGURE 1. BMP6 sequencing results. \#01: homozygous genotype for the BMP6 p.Gln158Ter pathogenic variant. \#02: heterozygous genotype for the BMP6 p.Val394Met genetic alteration. \#03: Heterozygous genotype for the BMP6 p.Arg257His pathogenic variant. 
TABLE 1. General and clinical data of the patients.

\begin{tabular}{|c|c|c|c|c|c|}
\hline ID & Age/sex & $\begin{array}{c}\text { TS }(\%) / \\
\text { and SF } \\
(\mu \mathrm{g} / \mathrm{L})\end{array}$ & LIC & $\begin{array}{c}B M P 6 \\
\text { variants }\end{array}$ & $\begin{array}{c}\text { Reference of } \\
\text { alteration }\end{array}$ \\
\hline \multirow{2}{*}{ \#01 } & 61 & $82 /$ & & p.Gln158Ter & \multirow{2}{*}{ Novel } \\
\hline & Male & 1,505 & & exon 1 & \\
\hline \#02 & $\begin{array}{c}46 \\
\text { Male }\end{array}$ & $61 / 1,096$ & & $\begin{array}{c}\text { p.Val394Met } \\
\text { exon } 4\end{array}$ & Novel \\
\hline \multirow{2}{*}{ \#03 } & 32 & 46.4 / & \multirow{2}{*}{$1.2 \mathrm{mg} / \mathrm{g}$} & p.Arg257His & Piubelli C., et \\
\hline & Male & 301.4 & & exon 2 & al.2017 \\
\hline
\end{tabular}

ID: identification; TS: plasma transferrin saturation; SF: serum ferritin; IO: Iron overload. Notes: for patient \#01 no changes in the HFE, HJV, HAMP, TFR2, SLC4OAl or FTL genes. In patient \# 02, no changes were found in the HJV, HAMP, TFR2, SLC4OA1 or FTL genes. Father, mother, 2 sisters with IO. In patient \# 03 no changes in the HFE, HJV, HAMP, TFR2, SLC4OA1 or FTL genes. Father was diagnosed with IO. 
TABLE 2. Population frequency, in silico prediction of alterations found in the BMP6 gene and classification of variants according to American College of Medical Genetics and Genomics (ACMG).

\begin{tabular}{|c|c|c|c|c|c|c|c|c|c|c|c|c|}
\hline \multirow[b]{2}{*}{ ID } & \multirow[b]{2}{*}{$\begin{array}{l}B M P 6 \\
\text { mutation }\end{array}$} & \multicolumn{2}{|c|}{ Population frequency } & \multirow[b]{2}{*}{ Provean } & \multicolumn{4}{|c|}{ In silico prediction } & \multirow[b]{2}{*}{ ClinVar } & \multirow[b]{2}{*}{ Change Type } & \multirow[b]{2}{*}{ ACMG criteria } & \multirow[b]{2}{*}{ ACMG } \\
\hline & & $\begin{array}{l}\mathrm{ABraOM} \\
(\%)\end{array}$ & $\begin{array}{l}\text { GnomAD } \\
(\%)\end{array}$ & & $\begin{array}{l}\text { PolyPhen- } \\
2\end{array}$ & SIFT & HGMD & $\begin{array}{l}\text { Mutation } \\
\text { taster }\end{array}$ & & & & \\
\hline \#01 & p.Gln158Ter & & & & & & & $\begin{array}{l}\text { Prediction } \\
\text { disease } \\
\text { causing }\end{array}$ & $\begin{array}{c}\text { not } \\
\text { reported }\end{array}$ & $\begin{array}{l}\text { Intronic } \\
\text { region }\end{array}$ & $\begin{array}{l}\text { PVS, PM2 e PP4, other criteria shown above are not } \\
\text { met }\end{array}$ & Pathogenic \\
\hline \#02 & p.Val394Met & 0.000821 & 0.0005129 & tolerated & benign & tolerated & & $\begin{array}{l}\text { disease } \\
\text { causing }\end{array}$ & $\begin{array}{l}\text { not } \\
\text { reported }\end{array}$ & Missense & $\begin{array}{l}\text { PM2. PP2 e PP4, other criteria shown above are not } \\
\text { met }\end{array}$ & VUS \\
\hline \#03 & p.Arg257His & 0.004926 & 0.001375 & damaging & $\begin{array}{l}\text { probably } \\
\text { damaging }\end{array}$ & deleterious & & $\begin{array}{l}\text { disease } \\
\text { causing }\end{array}$ & $\begin{array}{c}\text { not } \\
\text { reported }\end{array}$ & Missense & PM2. PP2, PP3, PP4 e PP5 & Pathogenic \\
\hline
\end{tabular}

PVS - pathogenicity very strong, PM - pathogenicity moderate, PP - pathogenicity supplementary, VUS - variant of uncertain significance 
Supplementary table 1. Primers, hybridization temperature for the application of exons of the BMP6 gene.

\begin{tabular}{|c|c|c|}
\hline Primer pairs & Sequence & A.T. $\left(C^{\mathbf{o}}\right)$ \\
\hline BMP6 1A F & GGGACTTGCTCACGCCAAGG & 59.6 \\
\hline BMP6 1A R & CGGACAGGGCGTTGTACAGAT & \\
\hline BMP6 1B F & CTCAAGACGCAGGGAAGCG & 61.8 \\
\hline BMP6 1B R & GGGAAATGTTCTCGTCATTACGTT & \\
\hline BMP6 2 F & ATCCACCTATGAAGTCAAACGGG & 56.7 \\
\hline BMP6 2 R & GGAGTTATCTGATCACAAAAGATGCTT & \\
\hline BMP6 $3 \mathrm{~F}$ & GGCATGTTTTATCCTGACGCTGAGA & 56.7 \\
\hline BMP6 3 R & GTCCTCCCATCGCCATCCTCTT & \\
\hline BMP6 4 F & AGGAAACTAGGAAGTATCCCTTAACTG & 63.4 \\
\hline BMP6 4 R & CTGAGTCTTCAGGTAACAAGTGTTC & \\
\hline BMP6 $5 \mathrm{~F}$ & GCTGTTGGGTGACCATACTTGTATC & 59.6 \\
\hline BMP6 $5 \mathrm{R}$ & GGCTTTGAAAGGATGAGGTGAGAAT & \\
\hline BMP6 6_7 F & CACATATTGCTGGCCAACCTACG & 56.7 \\
\hline BMP6 6_7 R & CCGAGATGGCATTTAGCTTAGTTG & \\
\hline BMP6 7 F & AACCAACCACGCGATTGTGC & 56.7 \\
\hline BMP6 7 R & GGAGGGTAGGAAGAGCTTCACGG & \\
\hline
\end{tabular}

A.T.: annealing temperature. 


\section{References}

1. Brissot, P., Pietrangelo, A., Adams, P. C., de Graaff, B., McLaren, C. E., \& Loréal, O. (2018). Haemochromatosis. Nature Reviews Disease Primers, 4, 18016.

2. Brissot P. Optimizing the diagnosis and the treatment of iron overload diseases. Expert Rev Gastroenterol Hepatol. 2016;10(3):359-70. doi: https:// doi.org/10.1586/17474124.2016.1119043.

3. Porto G, Brissot P, Swinkels DW, Zoller H, Kamarainen O, Patton S, et al. EMQN best practice guidelines for the molecular genetic diagnosis of hereditary hemochromatosis (HH). Eur J Hum Genet. 2016;24(4):479-95. doi: https://doi.org/10.1038/ejhg.2015.128.

4. Santos PC, Dinardo CL, Cancado RD, Schettert IT, Krieger JE, Pereira AC. Non-HFE hemochromatosis. Rev Bras Hematol Hemoter. 2012;34(4):311-6. doi: https://doi.org/10.5581/1516-8484.20120079.

5. Santos PC, Krieger JE, Pereira AC. Molecular diagnostic and pathogenesis of hereditary hemochromatosis. Int J Mol Sci. 2012;13(2):1497-511. doi: https:// doi.org/10.3390/ijms13021497.

6. L. C. Pilling et al., "Common conditions associated with iron overload genetic variants : cohort study in UK Biobank,” BMJ, vol. 364, no. k5222, 2019.

7. P. Brissot and O. Loréal, "Iron metabolism and related genetic diseases: A cleared land, keeping mysteries,” J. Hepatol., vol. 64, no. 2, pp. 505-515, 2016.

8. A. Pietrangelo, "Non-HFE hemochromatosis," Semin. Liver Dis., vol. 25, no. 4, pp. 450-460, 2005.

9. Pietrangelo A. Genetics, genetic testing, and management of hemochromatosis: ears since hepcidin. Gastroenterology. 2015;149: 1240-1251. 
10. Badar S, Busti F, Ferrarini A, et al. Identification of novel mutations in hemochromatosis genes by targeted next generation sequencing in Italian patients with patients with unexplained iron overload. Am J Hematol. 2016;91:420-425

11. Faria R, Silva B, Silva C, Loureiro P, Queiroz A, Fraga S, et al. Next-generation sequencing of hereditary hemochromatosis-related genes: Novel likely pathogenic variants found in the Portuguese population. Blood Cells, Mol Dis. 2016 Oct $1 ; 61: 10-5$.

12. Santos, P. C., Cançado, R. D., Pereira, A. C., Schettert, I. T., Soares, R. A., Pagliusi, R. A., ... \& Chiattone, C. S. (2011). Hereditary hemochromatosis: mutations in genes involved in iron homeostasis in Brazilian patients. Blood Cells, Molecules, and Diseases, 46(4), 302-307.

13. Bragdon, B., Moseychuk, O., Saldanha, S., King, D., Julian, J., \& Nohe, A. (2011). Bone morphogenetic proteins: a critical review. Cellular signalling, 23(4), 609-620.

14. Camaschella C. BMP6 orchestrates iron metabolism. Nat Genet. 2009;41:386388.

15. McDonald CJ, Ostini L, Wallace DF, et al. Next-generation sequencing:Application of a novel platform to analyze atypical iron disorders.J Hepatol. 2015;63:1288-1293.

16. Parrow NL, Fleming RE. Bone morphogenetic proteins as regulators of iron metabolism. Annu Rev Nutr. 2014;34:77-94.

17. Badar S, Busti F, Ferrarini A, et al. Identification of novel mutations in hemochromatosis genes by targeted next generation sequencing in Italian 
patients with patients with unexplained iron overload. Am J Hematol. 2016;91:420-425

18. Kautz L, Meynard D, Monnier A, et al. Iron regulates phosphorylation of Smad1/5/8 and gene expression of Bmp6, Smad7, Id1, and Atoh8 in the mouse liver. Blood. 2008;112:1503-1509.

19. Kautz L, Besson-Fournier C, Meynard D, et al. Iron overload induces BMP6 expression in the liver but not in the duodenum. Haematologica. 2011;96:199203.

20. Meynard D, Kautz L, Darnaud V, et al. Lack of the bone morphogenetic protein BMP6 induces massive iron overload. Nat Genet. 2009;41:478-481.

21. Andriopoulos B, Corradini E, Xia Y, et al. BMP6 is a key endogenous regulator of hepcidin expression and iron metabolism. Nat Genet. 2009;41:482-487.

22. Piubelli, C., Castagna, A., Marchi, G., Rizzi, M., Busti, F., Badar, S., ... \& Suku, E. (2017). Identification of new BMP6 pro- peptide mutations in patients with iron overload. American journal of hematology, 92(6), 562-568.

23. Canali, Susanna, et al. Endothelial cells produce bone morphogenetic protein 6 required for iron homeostasis in mice. Blood, 129.4 (2017): 405-414.

24. QIAGEN, Inc. Manual do Mini Kit DSP DNA de sangue QIAamp®. Hilden: QIAGEN, 2004, 1-27. . In.

25. Kobayashi Y, Yang S, Nykamp K, Garcia J, Lincoln SE, Topper SE. Pathogenic variant burden in the ExAC database: an empirical approach to evaluating population data for clinical variant interpretation. Genome Med. 2017;9(1):13.

26. Richards S, Aziz N, Bale S, et al. Standards and guidelines for the interpretation of sequence variants: a joint consensus recommendation of the American 
College of Medical Genetics and Genomics and the Association for Molecular Pathology. Genet Med. 2015;17(5):405-424.

27. Piubelli, C., Castagna, A., Marchi, G., Rizzi, M., Busti, F., Badar, S., ... \& Suku, E. (2017). Identification of new BMP6 pro- peptide mutations in patients with iron overload. American journal of hematology, 92(6), 562-568.

28. Daher R, Kannengiesser C, Houamel D, Lefebvre T, Bardou-Jacquet E, Ducrot N, et al. Heterozygous Mutations in BMP6 Pro-peptide Lead to Inappropriate Hepcidin Synthesis and Moderate Iron Overload in Humans. Gastroenterology. 2016 Mar 1;150(3):672-683.e4.

29. McDonald CJ, Rishi G, Secondes ES, Ostini L, Wallace DF, Crawford DHG, et al. Evaluation of a bone morphogenetic protein 6 variant as a cause of iron loading. Hum Genomics. 2018 Dec 25;12(1):23.

30. Hamdi- Rozé, Houda, et al. "Rare HFE variants are the most frequent cause of hemochromatosis in non- c282y homozygous patients with hemochromatosis." American journal of hematology 91.12 (2016): 1202-1205. 